\title{
News, Trends and Comments
}

\author{
NEWS
}

\section{STN's New Databases}

STN announced five new databases in November. PATGRAPH contains the graphic images from the front pages of recent German patent applications. NBSFLUIDS (produced by the US Department of Commerce National Institute of Standards and Technology) contains a program MIPROPS, that calculates thermophysical and transport properties over a wide range of temperatures and pressures for 12 cryogenic fluids. PHYTOMED is a German bibliographic database (produced by Biologische Bundesanstalt fuer Land und Forstwirtschaft) on the world literature of plant sciences. FSTA (produced by the International Food Information Service) covers information from 1800 of the world's food science and technology journals, and contains 120,000 citations from 1981 onwards. LCASREACT is a training file for STN's CASREACT reaction search file. For more phone CAS Customer Service 800-848-6538, or write STN c/O CAS Customer Service, PO Box 3012, Columbus, OH, 43210 USA.

\section{Electronic Publishing International Conference}

The 9th Annual Conference of the International Electronic Publishing Research Centre (IERPC) Ltd., will be held at the Amsterdam Marriott Hotel, The Netherlands, on the 25th-27th April, 1990. IERPC is a nonprofit institute for research on electronic technologies in the publishing process. The session Authors note: These pages have been produced on a new word processing systen. By the next issue the new toy syndrome will have evaporated so the effect should aesthetically be nore pleasing headings are Publishing and applications of EP; New media in publishing; Non-conventional and corporate publishing; European issues. Speakers are drawn from a variety of organisations from Europe and the United States including Dow Jones, Hachette, the British LIbrary, Price Waterhouse, Apple, Sony, CEC DG XIII and several others.

The attendance price is $£ 390$ for delegates, and $£ 490$ for nondelegates. For more phone 44372376161 , or write Penny Young, Status Meetings Ltd., Festival Hall, Petersfield, Hants, GU31 4JW, England. 


\section{ADONIS}

Following a two year trial, ADONIS is scheduled to become operational on January 1st 1991. It has been demonstrated that cost savings can be made compared with conventional photocopying, and that the ADONIS identifier, a number allocated at the time of indexing, is superior to other methods of uniquely identifying an article.

The service will be medically predominant, but other fields including Agriculture, Biology, Chemistry, Engineering, Pharmacology, Physics, Public Health and Veterinary Medicine are being considered.

Tests have shown that retrieval and printing from an ADONIS CDROM and workstation can produce cost savings of up to $50 \%$ compared with the costs associated with conventional photocopying. There may be other significant savings such as shelving economies, binding costs, etc.

Nearly 200,000 articles were scanned and stored during the trial up to May 1989.

\section{CAB Abstracts on CD-ROM}

$\mathrm{CAB}$ announced in September that it will publish its CAB Abstracts and the abstract journal series based on them from 1984 onwards on CDROM. This database will be supplied in two volumes - 1. 1984-1986 inclusive; 2. 1987-1989. CAB decided to use the Silver Platter Information Retrieval System (SPIRS) after many months of testing. It will be possible to store nearly 400,000 abstracts on one disc. To run a disc, an IBM PC or compatible with 640K RAM, MS-DOS or PC-DOS 2.1 or higher, and a CDROM player will be needed.

For more phone 049132111 in the UK or 8005284841 in the US, or write $\mathrm{CAB}$ International, Wallingford, Oxon, OX10 8DE, England, or CAB International, 845 North Park Avenue, Tucson, Arizona 85719, USA.

\section{Change of Director at ASLIB}

Dr. Dennis Lewis retired in October and Mr. Roger Bowes was appointed as the new Chief Executive on December 1st.

Dr. Lewis "changed Aslib's role from that of a cosy club to its current position as the foremost organisation in the United Kingdom promoting the concept that information is a corporate, organisational and social resource requiring the development and use of specialist skills". He will now be working with the European Commission.

Roger Bowes was Chairman of Citybridge, Management Consultants, and previously Chief Executive of Express Newspapers plc. Mr Bowes has been active on several committees and councils in the UK publishing and information industry.

\section{Chemical Abstracts to co-operate with USSR Academy of Sciences}

In October Chemical Abstracts announced the signing of a protocol of intent to cooperate with the USSR Academy of Sciences and State Committee for Science and Technology. The Academy of Sciences is a government financed system of scientific institutes engaged mainly on fundamental research. The State Committee guides research, establishes priorities, and provides funds.

CAS said that their main interest would be in ensuring prompt access to USSR scientific and technical information. 


\section{NFAIS News}

The National Federation of Abstracting and Information Services announced in September that its membership had reached an all-time high, having increased by $20 \%$ in 1989. New members include NewsNet, Predicasts, Silver PLatter, and Towers Perrin. At the same time NFAIS announced that it will be publishing four new reports: Information Industry Terms \& Conditions; Secondary Information Service Statistics; Economies of Database Production; Global Copyright Issues in the Secondary Information Industry.

More information from Lee Gros, 215563 2406, or write to NFAIS, 1429 Walnut St., Philadelphia, Pa., 19102, USA.

\section{Library Automation Conference}

The first European Conference on Library Automation \& Networking will take place in Brussels on 9th-11th May, 1990. An exhibition and a three way videoconference between European, American, and Japanese librarians is included. The conference will include sessions on New Tools; The CEC draft Action Plan for Libraries, Networking between Publishers, Distributors, and Libraries; New Technologies and Data Collection for Preservation; Librarianship - a changing profession in a changing Europe: Videconference.

For more phone Brussels 3226476060 , or write to A.M.M., Rue de Trone 215, Troonstraat, B-1050, Brussels, Belgium.

\section{Accelerator for IBM PS/2 machines}

In November IBM announced the PS/2 Wizard Adapter card, with OS/2 software developer tools and optional memory expansion. The card incorporates the Intel i860 Reduced Instruction Set Computing (RISC) processor. Co-processor power has become a feature of present generation microcomputers. Computer-intensive tasks are taken over by a computer within a computer, freeing the main processor for a wider range of general tasks.

Among other features the i860 contains a special graphics processing unit. Image processing and graphics are advancing steadily thanks to chip power and high-resolution monitors $(2000 \times 2000$ pixels or more) at prices which have dropped to about one quarter of their prices when first introduced three or four years ago.

The Wizard card comes with 2 Mbytes of 85 nanosecond DRAM. The 64 bit processor runs at $33 \mathrm{MHz}$ and the Wizard is capable of 64 Million Floating Point Operations Per Second (MFLOPS). It has been demonstrated creating fractal images at 33 MFLOPS, and performing seismic data processing operations at 55 MFLOPS. Such power does not come cheap The Wizard Adaptor will be available in 1990 at £5568. An extra 6 Mbytes of DRAM costs $£ 2915$, and software tools $£ 1168$.

Our information came from IBM UK 0705 321212, at PO Box 41, North Harbour, Portsmouth, Hants PO6 3AU, England.

\section{British Telecom launches ISDN 2}

BT announced this service in November under the misleading title "Launch of the digital communications era". ISDN is another, albeit potentially important step, in digitising the telephone network. Digitisation 
began many years ago and is nearing completion within most national and international telephone networks.

Digitisation of the telephone exchange network has been carried out to obtain various engineering advantages and also to enable a relatively small number of computer-like store-and-forward semiconductor switches to be installed as a replacement for a larger number of assorted types of earlier generation devices in exchanges.

The part which has not been digitized is the largest part - the "local loop". From each exchange thousands of copper-pair wires radiate out to subscribers and nearly all of them remain as they have been for many years.

The ISDN - an idea formulated by the Post and Telephone Authorities (PTTs) in the late seventies - will complete the digitising process by the installation of equipment at either end of the exchangesubscriber wire. The enormous cost of replacing the local loop has been put off by limiting the basic local ISDN bit rate to 140 Kilobits per second (Kbps). Most of the local loop in all countries will carry data at this rate. Copper pairs will be gradually replaced by fibreoptic cable enabling the bit rate to be speeded up so that the transmission of items such as images in colour will become feasible.

Eventually all subscribers will use telephones which digitise their voice and convert their caller's voice back to sound waves. They will also be able to plug in a data terminal in order to use a range of new services. Digitised voice from the telephone will run at $64 \mathrm{Kbps}$, and so will the data channel, leaving a little spare capacity in the local loop for a separate channel carrying control signals called the "D channel".

This control channel, allowing a far wider range of data than is sent within the speech channel of the conventional telephone network, will carry the signals controlling a range of alternative services.

British Telecom has been running an experimental Integrated Digital Access (IDA) service, a form of pilot ISDN, for some time and has just moved towards a full service by ordering an exchange capable of supporting 90,000 ISDN lines "allowing BT's public telephone network to meet our customer's communication needs for data, text, fax, graphics, and video, as well as voice, over a single high-speed digital connection". Other countries are at various stages of network digitisation and ISDN implementation.

For single line IDA, BT charge $£ 500$ for connection and $£ 500$ a year rental. This provides a subscriber with two sockets into which may be plugged a digital telephone and a data terminal. One way of providing a terminal is to buy a communication board or card, costing about $£ 1000$ in the UK, and plug it into a PC. The PC can then transmit data at $64 \mathrm{Kbps}$ from the X21 interface (data terminal to synchronous public network circuit terminating point) on the card. However this may result in some unexpectedly slow, and therefore unexpectedly expensive transmissions because of the PC's limited processing and protocol handling power.

The "arrival" of the ISDN - meaning the connection of a significant number of subscribers in any country - will be determined by the allimportant chicken-and-egg factor; applications may burgeon once some critical mass of subscribers are connected, but they won't connect until they can see the benefits.

Other reasons for delays include ratification of an important standard - CCITT Q.932 - perhaps in 1991. This standard covers signalling protocols to be used in the "D" channel. Formulation, agreement, and ratification of standards always lags behind the commercial imperatives of 
getting on with it, possible loss of competitive advantage, etc. The game is to get going with a "pre-standard best guess" implementation which is as effective as possible and near enough to the expected standard to be modifiable later without too.much delay or difficulty. When Q.932 becomes agreed, software has to be written and tested.

Subscriber/PTT installation costs reside mainly in the local interfaces and Network Terminating Equipment (NTE), and in special terminals. The cost of these items will be much lower if their functions are performed by chips. Chips must be produced in volume to get prices down so it is risky to jump the gun on standards. If it is jumped (as has been done already in the United States) with a bad guess, the less inclined will be the jumpers to conform to the standard when it arrives, and the greater the delay in its adoption.

In 1987 the Bell Company, US West, listed customers such as Honeywell, US National Bank, PNB, and others, participating in their ISDN trials. 40 different applications were being tested. According to US West, "For controlling and managing the size and the cost of the network... the ISDN can impact the business customer"... although the market opportunity... may not translate easily from existing user applications".

At present in the UK it seems that there is little incentive to use ISDN lines and facilities. The large organisations who have the resources to organise purpose-designed private networks using leased lines do not feel that ISDN has much to offer that is better.

There has been some interest in "Multiline IDA" where blocks of $3264 \mathrm{Kbps}$ lines are available. One application is in improving PBX connections. This seems to point to the benefits of digitisation rather than to the ISDN.

From about 1995 onwards Broadband ISDN is expected to appear. The available bandwidth will be wider. Faster speeds and lower (presumably) costs will encourage the transmission of pages' of text and images in colour.

\section{Online Information 89}

$$
\begin{array}{r}
\text { The spirit of the time shall teach me speed } \\
\text { Shakespeare (King John) }
\end{array}
$$

The 13th annual meeting and exhibition was held in London on the 12th-14th December 1989. The papers are covered in Online Information 89: 13th International Online Meeting, London 12-14 December 1989. Published by Learned Information, Oxford \& New Jersey, 1989. ISBN 0 904933725 . Price $£ 30$.

Last year the emphasis was on Hypermedia, CD-ROMs, and Expert Systems. It still is.

\section{Hypermedia and images}

M.Percival and N.MacMorrow (University of Strathclyde) consider the virtues of the Mac (computer, that is) in "Evaluating the feasibility of using Hypercard as an interface prototyping tool with reference to online services: the impact of ISDN". The ISDN bit is accounted for because British Telecom, sorely in need of ISDN applications (as noted in an earlier section), funded the project.

The Mac screen presentation is the best of its type so it makes sense to offer it to a user as an interface to an electronic mail system (Telecom Gold). Otherwise the user has to "enter long and complex codes at various stages..." The scripts associated with screens (or Hypercards), 
written by the designer and unseen by the user, are responsible for providing the easy access to the system. One reason for using it on the ISDN would be the speed with which images could be transmitted.

The Hypermedia-image connection comes up again in "A Hypermedia structure for an interactive and progressive image retrieval system" by G.Halin and C.Hamon from CRIN, France. The objective is to "permit interactive and progressive browsing in an image database".

The problem is how do you index an image? Halin and Hamon tackle it by providing a thesaurus with sub-divisions representing domains of knowledge. The system aids the user to express his request through successive refinements. "Expressivity weights" are allocated to terms according to the user's assessment of their ability to express the demand. Links between images in a hypertext structure are added to chart the user's course. The software, called RIVAGE, which runs on a sun workstation, is based on Smalltalk, and is used with a videodisc machine which carries the image database.

This sounds like a very good approach to solving a problem which badly needs a solution.

\section{CD-ROMs versus Online}

In "CD-ROM versus online: an evaluation of the effect of the user interface on search effectiveness - a pilot study", J.E.Rowley (Manchester Polytechnic) finds in favour of CD-ROM - at least when searching the BNB database. Searchers took the same or less time with CD-ROM and most found it easier to use. What we want to know as well, but are not told, is what about the costs? One comment helps in this respect "Testers were conscious that they were spending money with access to BLAISE, yet had the leisure to experiment with $\mathrm{CD}-\mathrm{ROM}$ at their own pace, and this may put testers under pressure to work faster when searching online".

In carrying out tests on the CD-ROM version of the SCI recently (See Cawkell, A.E., The Electronic Library 7(6), 345-350, December 1989 "Automatic indexing in the Science and Social Science Citation Index CDROM") I was very conscious that I was not incurring telecoms and online search costs. If I had been, would I have got through the job faster, thus saving labour/overhead costs? The balance-of-costs answer depends on the environment. It depends upon whether the most expensive item - the cost of a particular searcher's time is taken into account (presumably more likely in a commercial environment).

Expert systems to help with search strategies

There are two papers devoted to the same area of investigation in information retrieval:- "MOSS: a prototype expert system for modifying online search strategies" by A.Morris and others from Loughborough University, and "A knowledge base for the search profile analysis and user guidance" by E.Sormunen, Technical Research Centre of Finland.

Both are to do with the modification of search strategies by helping to improve the basic failures "Retrieved sets are too large", "Retrieved sets are too small" or "Retrieved sets are off-target". The "MOSS" paper starts with a lengthy review of previous attempts with 27 references. Prior to building, "all knowledge bearing statements had to be identified from protocol and interview transcripts and expressed as production rules". In the paper by Sormunen, lists of operational and conceptual moves are provided to show how search tactics may be changed as a search progresses.

"MOSS" seems to be ahead at the moment as claims are made for its success in practice. Sormunen says that "the quality of rules has not been evaluated in comprehensive field tests". 
Revival of Reaction Indexing... with the help of Transputers The articles about fashionable topics selected for mention above are joined by something of a revival in a much older topic; there are five papers about Reaction Indexing. This subject with its difficult classification problems, was pioneered by Weyl in his massive work Die Methoden der Organische Chemie, published in the first decade of this century. Its successor, Houben-Weyl's 32 volume Methoden der Organische Chemie, an encyclopedia, was started in 1952 and is, I believe, still incomplete. The main problem is simply the effort needed to keep it up to date.

An alternative method, introduced by Weygand, is probably better known, since it was adopted in a modified form by Theilheimer in the widely used Synthetic Methods of Organic Chemistry. Since 1980 Theilheimer has become a part of the Journal of Synthetic Methods, published monthly in machine readable form by Derwent. It includes coverage of patents.

The difficulties of classification in this field arise because relevant aspects to a chemist who wants to synthesise an end product as easily as possible and with a high yield, include starting materials, reaction products, reacting and formed bonds, reagents, conditions, and so on. Computerised systems are able to handle these various concepts together and help the chemist devise suitable strategies.

Papers by S.R.Heller, Dept. Agriculture USA and Scitechinform UK/USSR, and R.C.Dana, Chemical Abstracts, respectively, describe the coverage and compilation of the available reaction databases. Gasteiger and Weiske from the Technical University, Munich, describe the Fiz Chemie reaction database.

Dana divides users into end users, information professionals, and patent searchers. End users want a few good leads and often ask questions based on a small substructure. Information professionals know how to ask detailed questions and refine large answer sets; they tend to be concerned about inclusiveness. Patent searchers want all reactions from a given patent to be included in the database - as provided, for instance, in the IDC GREMAS database.

The papers most likely to be of interest to chemists are probably those describing current retrieval techniques. Although Shakespeare probably did not have the Transputer in mind when referring to "The spirit of the time" (See the quotation heading this section), the quotation is apt. The Lynch, Willett, et al team - associated with chemical structure searching for years - have now brought its speed to bear on this problem.

In "Parallel processing techniques for information retrieval: searching for textual and chemical databases using transputer networks", Cringean, Lynch, and others describe the current investigation. In order to gain the maximum advantage, the game is to spread the computing load so that all the Transputers are fully occupied all the time.

In searching 50,000 structures using 14 typical substructure queries, the ideal of a speed increase directly proportional to the number of Transputers in the network held good up to 10 Transputers. For 21 Transputers, the speed increase was 15 times. Cringean et al also report results obtained when serially searching document databases - a return to an old idea discarded in favour of inverted file searching in the days of slow processing.

They conclude "The results... demonstrate clearly that the Transputer provides an extremely cost-effective way of increasing the 
performance of microcomputer-based retrieval systems. However it is also clear that data transmission costs can severely constrain the level of performance that can be obtained in practice".

Other papers about this subject include "Reaction centre searching and fragment similarity searching using the ORAC program" described by G.A.Hopkins; P.J.McHale, Molecular Design Ltd., discusses the REACCS database management system and lists the databases that it may be used with.

\section{VIEWS AND COMMENTS}

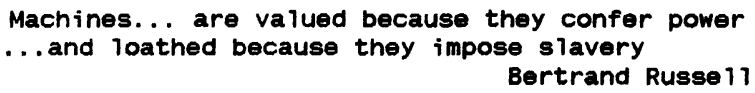

\section{Word Processing re-visited}

Russell got it nearly right. For current WP machines and software the imposition of slavery is accompanied by the imposition of undue complexity and useless instruction manuals.

The Power and the added facilities are certainly there. Having graduated from Sol with Word Wizard, via Vectorgraphic 4/30 with Memorite, to PS $/ 2$ with Word Perfect 5.0 , I have found that this is very obvious. Some comparative information is given in the box. The price of the PS/2 system complete with all software shown is about one quarter that of the Vector-

\begin{tabular}{|c|c|c|c|}
\hline MACHINE & Processor Technology & Vectorgraphic & IBH \\
\hline & Sol computer & $4 / 30$ computer & PS/2 30-021 computer \\
\hline IVP SOFTWARE & Word Wizard & Menorite & Word Perfect 5.0 \\
\hline PROCESSOR & 8080 8-bit 2HHz & 280B 8-bit 5HHz & 8086 16-bit 8HHz \\
\hline OPERATING SYS. & $\quad \quad C P / N 80$ & $C P / N 80$ & $\operatorname{Dos} 4.0$ \\
\hline MEMORY & $64 K$ & $128 k$ & $640 \mathrm{~K}$ \\
\hline OISCS & $2 \times 250 \mathrm{~K}$ & $680 x+5 k$ & $680 K+20 K$ \\
\hline PRINTER & Diablo daisywheel & Diablo daisywheel & Epson 24-pin matrix \\
\hline $\begin{array}{l}\text { WP, DATABASE, } \\
\text { OP.SYS. SIZE }\end{array}$ & $350 k$ & $800 k$ & $7.2 M$ \\
\hline
\end{tabular}
graphic in real

terms. The Memorite software designed around 1980, is far'more compact and less complex than Word Perfect. All the facilities needed are there including a very good speller. Some are better. For example although Word Perfect has all kinds of options for text deletion, the one I found most useful in Memorite was "delete up to...". You pressed one key and then typed a symbol or word. On pressing "Return" the text between the cursor and the symbol or word were deleted and the gap was closed up. This is not provided on Word Perfect.

What you get in Word Perfect is a six times increase in software size and about the same increase in complexity for a range of benefits some are bells and whistles, some are fringe benefits, and some are valuable. You also get a speed increase which in many instances is greater than the raw speed ratio increase. The product of processor speed and word length for the PS/2 is about 3.2 times that of the Vector; this provides a rough indication of expectations. In fact differences in file organisation, wait states and disc accesses probably at least doubles that difference. Accordingly the user's waiting time when copying files, printing, etc., is considerably diminished with Word Perfect. 
Word Perfect benefits include:-

1. the provision of utilities enabling sequences of operations to be carried out automatically instead of step by step.

2. Entirely new features.

3. Features providing improvements in the appearance of pages.

4. Certain attributes normally only found in a Desktop Publishing System.

An example of Item 1. is the auto-reference feature. If you have already typed, say, 25 superscript numbers at various places in the text, keyed to numbered references at the end, you may want to insert a missed reference - say between 11 and 12. This is normally a nuisance. You have to increment all the subsequent numbers by one at each place in the text, type in the new reference 12 in the reference section at the end in the right place, and increment all subsequent numbers in the reference section by one.

In Word Perfect this process is automatic. You insert a new superscript number 12 , press a key, and type out the new reference 12 on the blank screen which appears. Press another key and you are back in the text at superscript 12, all subsequent numbers having been incremented by one. Meanwhile the new reference 12 has been inserted in the right place in the reference section and all subsequent numbers in that section having also been incremented by one.

The box on the previous page is an example of Item 2 - an entirely new feature. The in-box text was first composed in a separate file. It was then shrunk by specifying Sans Serif 20 char/inch font instead of the normal Roman 12 point proportional. The file containing this News and Views article was recalled, the cursor placed at "The Power and...", and the text box menu called up. Menu items specifying the name of the file to be called into the box, the position of the box relative to the cursored paragraph, the dimensions of the box, and the requirement that the external text should be wrapped round it, were completed.

Decorative functions such as border and caption style, grey background infill, etc., may also be ordered as needed. Graphics may be retrieved into a box and scaled or rotated to fit if required. The figure just above shows a simple graphics file retrieved into a box with a double-line border, filled $20 \%$ grey scale. Upon exiting the menu back to the main text file, the box is visible in the specified position with wrapped text, but not its contents. But by using the "view page" key, the whole page just filling the screen, or segments of it enlarged, may be viewed, including the contents of boxes, showing different fonts, lines etc., black on paper-white exactly as they will be printed. Final editing - e.g. symmetrical text infill of box adjustment - may be carried out as necessary. As an example of Items 3 and 4 , note the previous two pages, which include three fonts (the text being in a proportionally spaced font), boxed text, and boxed graphics with infill.

A user's expectations in view of the complexity increase of today's systems would be a corresponding increase in the amount and clarity of explanatory material. Unfortunately most of the material supplied with the computer, the operating system software, and the WP software shows that 
little attention has been paid to the most elementary instructional and indexing principles. The manuals have obviously not been tried out on a consumer before publication.

The manual supplied with the PS/2 contains no specification, and no list of functions, block diagram, general overview, performance data, memory size, or disk capacity. Nothing is said about the screen resolution or image facilities. The dealer registered a blank stare when I asked him about the screen - EGA? MGA? MCGA?. He had no idea.

To set up the system so that it starts up as required, it is necessary to enter data into a file called AUTOEXEC.BAT. The DOS manual provides the following example, and I quote "...of what you might find in the AUTOEXEC.BAT file" :-

\section{@ECHO OFF SET COMSPEC $=\mathrm{C}: \backslash$ DOS $\backslash$ COMMAND.COM VERIFY OFF \\ PATH C: \DOS APPEND/E APPEND C: $\backslash D O S$ PROMPT \$P\$G \\ C: \DOS\GRAPHICS VER DOSSHELL}

Should commands of this kind always be present, or only some of them? Which are mandatory? Are any mandatory? Say we look up APPEND in the index - it's there - on page 138, where it says:-

"APPEND Displays a list of appended paths, if you use APPEND without any parameters after it is loaded. (After APPEND is loaded, it is an internal command.

Note that these "instructions" consist of a list of useless
statements.

A single example will have to be enough to show the kind of thing you are up against with the Word Perfect manual.

To handle a box two kinds of operation are required - first, create the box and specify its position, second deal with the "options" such as borders, infill, etc., - and that is the order of doing it as shown in the book. In fact you have to set up the options first - i.e. deal with the cosmetics of the box before you create it!

Though this be madness, yet there is method in it Hamlet, Act 1

....but can you see the method?

It is not clear whether suppliers of these systems are selling to computer professionals, large corporations, small businesses, authors, or whoever. Whatever the marketing objectives, the instructional materials are a failure for any of these markets.

\section{The Things they write about}

People publish some very odd articles in scientific, social science, and technical journals. Why should Mr Donato want to tell us about "A direct method for the propagation of error using a personal computer spreadsheet program" (J. Chem. Ed. 65(10), 867, Oct 1988)? Measurements in science are all-important but who needs "A simple microcomputer interface for tail-flick determination"? D.P.Harris tells us all about it in J.Pharm.Met 20(20), 103, Sept. 1988. But computer programmers can behave almost like humans - they can even assume a kind of old worlde charm. 
C.L. Muehlenhard writes in the Journal of Computer Based Instruction (15(1), 7, 1988) about "Helping women to break the ice - a computer program to help shy women start and maintain conversations with men". Presumably shy women are supplied with a kind of head-up display, like they have in military aircraft, built-in to their glasses.

Mr R.Kok thinks you may be interested in a program for the Macintosh called McDrain. Read Canadian Agr. Eng. 30(2), 195, July 1988, for - "McDrain: a surveying, mapping, and drainage design system for the microcomputer". My micro doesn't get waterlogged - does yours? However for really getting to grips with the sociology of drainage, get the February 1989 issue of Plains Anthropologist and read about D.D.Scott's examination of "An officer's latrine at Fort Larned and inferences on status".

This reminds me of the need to publicise an historical mistake. Victor Allen did some research on the invention of the Water Closet and reported his findings in the New Scientist (24th December 1987). It was not invented by Thomas Crapper. Sir John Harrington (1561-1612) wrote a paper about it much earlier. He described the primeval WC in some detail in "Metamorphosis of Ajax; A Cloacinean satire". Sir John might well have said in his article "Queen Elizabeth sat here", although this is not confirmed by Allen. What Allen does suggest is that "had the uncouth elements of today a greater interest in history, they would speak of "going for a Harrington"".

What is the difference between Black Smokers and White Smokers? I can tell you - Black Smokers are fuelled by freezing magma. What is the fellow talking about, you may well ask. Read J.R.Cann's article in Nature 298, July 8th, 1982 for a complete answer. It turns out that a Black Smoker is something horrid exuding iron, zinc, and sulphides in volcanic areas like the East Pacific: Rise.

Now here's a point of some interest; most people don't know this. "Hypogonadotropic hypogonadal men respond less well to androgen substitution treatment than hypergonadotropic hypogonadal men" (Louis Gooren in the Archives of Sexual Behaviour 17(3), 265, June 1988). If you have spotted the difference and have recovered from this dreadful revelation, pass on to something more polite and ponder the importance of "The effectiveness of Chinese print advertisements in North America" ( $\mathrm{J}$. Advertising Res. 28(5), 25, Oct. 1988).

\section{SHORT REVIEWS OF BOOKS}

Manual of Online Search Strategies. Edited by C.J.Armstrong and J.A.Large. Gower Publishing Co., Aldershot, England. 1988. ISBN 0566 05058 7. 831 pages. Price $£ 45$.

This must be the meatiest book published in this field. There are a number of chapters about different subjects - Engineering, Law, Humanities, Health Sciences, Biological Sciences, Chemistry, and so on, each written by an author who specialises in that subject. These chapters follow more or less the same pattern providing a description of the major databases covering the area followed by numerous examples of worked searches often emphasising special features of databases.

Special topics have their own chapters. Chapter 1 provides general guidance on developing search strategies. Chapter 2 covers citation indexing with a description of the unique arrangement of the printed 
volumes leading to examples of how it may be used online. The specialised nature of Patent searching also justifies a special chapter which includes advice about where and how to search for patents in specific subject areas.

The last three chapters are also about special topics "Systems and databases for office and home use", covering Electronic Mail and databases claimed to user-friendly (are there any which aren't?), "Databases for quick reference" such as news databases, and "Electronic Journals". Nearly all chapters in this book are well referenced.

The book ends with a 50-page list of databases and their hosts, a small list of directories, and a comprehensive index. This manual should be within easy reach of anyone who is engaged in online searches covering different subjects on different host computers.

Geography of the Information Economy. Mark Hepworth. Belhaven Press, London. 1989. ISBN 185293029 2. 258 pages. Price $£ 27.50$

This book tackles information from a different angle - the geographical distribution of the information economy. A suitable sub-title would be "The politics of information distribution". Mr. Hepworth works at the Centre for Urban and Regional Development Studies in the University of Newcastle, UK.

In the preface it is suggested that "the spread of Information and Communication Technology in conjunction with the "informatisation" of the economy carries with it implications for the location of activities and the development of cities and regions as profound as the spread of railways, roads and electric power". Most of the implications seem to be rather unpleasant.

Having discussed computer networks in multilocational firms, aspects of "flexible manufacturing" and "just-in-time" techniques heavily dependent on immediate accurate information - Hepworth concludes that "it is incumbent on researchers to specify... the "uncertainty factor" which currently operates dramatically to change the social division of labour".

With regard to the UK it is concluded that "Entrepreneurism is replacing managerialism... A major result of this ideological shift, under the Thatcher administration, is increased economic competition between "entrepreneurial cities".... but job opportunities outside the information sector appear to be restricted to lower order service occupations. These class inequalities in production are importantly converging with emerging inequalities in consumption... threatening to undermine the local welfare state and possibly local democracy".

Continuing in the same vein, the growing informational activities of local authorities "...has more to do with the political "New Right" ideology of the present Thatcher administration", and in the book's concluding words "the ascendancy of the information worker... and the disappearance of the old factory worker... go hand-in-hand with the rest of the labour market propped up by an abundance of "flexible job" opportunities, a dismal array of lower order service occupations, and in the UK at least, by the Thatcher government's numerous training schemes".

I don't recall having seen this kind of political comment about the much discussed "Information Society" before. Herbert Simon, a well known figure in sociotechnology, expressed alternative views some years ago in a more detached manner (Science 203, January 12th 1979, 143-147):- 
"Automation and computerisation... eliminate mostly jobs that were already relatively routine... while service workers, sales personnel, and professional and technical workers increase, there will be a net increase in reported job satisfaction. Empirically, we find no signs of a downward trend in work satisfactions, and when we look at the actual impact of automation upon the workplace and the work force, we find no reason why such a trend should be expected. On the contrary, the newer technologies may even have a modest humanizing effect on the nature of work".

Conferences and their literature: a question of value. Robert Oseman. Library Association, London. 1989. ISBN 085365748 3. 146 pages. Price $£ 19.50$.

In the foreword of this publication, Maurice Line says "There are good reasons why people go to conferences. They are much better than work... You realize that other delegates are no more competent than yourself and thus you feel less inadequate, or better, superior. You meet people - clever people, useful people, nice people you would never come in contact with through the published literature; you also meet unpleasant people and develop skills in avoiding them at subsequent conferences".

Line adds "For these reasons I am convinced that teleconferencing will never catch on seriously (tele-meetings might, but that was said 10 o]. 20 years ago). Even bad and boring papers can be turned to advantage; I have drafted papers of my own while bored or when infuriated into reaction - what a colleague calls the manurial value of rubbish".

The book does not contain much about the value of conference literature. "Conference proceedings are erratic in both their editorial and their publishing style, as well as in delays in publication, placing considerable difficulties upon any library organisation that attempts to collect them". $10 \%$ of requests received by The British Library Document Supply Centre are for conference items and they are able to satisfy $94 \%$ of them. The number of proceedings received by the BLDSC has gone up from about 4000 in 1965 to nearly 20,000 in 1987. J.L. Head's attempt to assess value by citations received is discussed, but the study was too small for useful conclusions. The author discusses ways of pursuing this method.

It is disappointing that there is so little about value, but there is a great deal of other interesting information. Evidently about $65 \%$ of all organisations publish proceedings of their conference in full, $55 \%$ in special publications and $45 \%$ in journals. $64 \%$ of the published papers will have been peer reviewed, but in the author's survey it was not possible to estimate overall delays in publication. Other studies show great variations - for example following meetings by various US bodies, publication completion varied from $22 \%$ to $62 \% 18$ months later.

Here are some other interesting extracts:- "... sometimes a sudden acceleration in research activity may prove too rapid for the slow editorial and review procedures of conventional journal publication... conferences provide the opportunity for research workers to announce and discuss developments in timely fashion". The first announcement of new developments in high temperature superconductors came in September 1986; the rate of progress made it essential to hold an early conference. Five thousand scientists tried to attend a meeting arranged for April 1987. Twelve hundred got in and the scheduled evening session ended at $4 \mathrm{a.m}$. There is much of interest in this book, including a very useful chapter about growth patterns and exponential functions. 